\section{Dos esgrafiados y dos estampillas procedentes del yacimiento Dehesa Cintruénigo III (Tarazona, Zaragoza)}

$$
\begin{aligned}
& \text { Two graffiti and two stamps } \\
& \text { found in Dehesa Cintruénigo } \\
& \text { III (Tarazona, Zaragoza) }
\end{aligned}
$$

\section{Carlos García Benito \\ Centro de Estudios Turiasonenses \\ carlosgarciabenito@gmail.com \\ María José Estarán Tolosa \\ Universidad de Zaragoza \\ mjestaran@unizar.es \\ Julián Pérez Pérez \\ Centro de Estudios Turiasonenses \\ julianperezperez@hotmail.com}

Romina Luesma González
Centro de Estudios Turiasonenses
rominaluesma@gmail.com
José Ángel García Serrano
Centro de Estudios Turiasonenses
jykdos@gmail.com

Resumen: El yacimiento Dehesa Cintruénigo III (Tarazona, Zaragoza) se corresponde con un antiguo lagar que comprende una zona de pisado sobre un lacus de tipo trullo, los restos de una prensa de viga manual de tipología heroniana y un espacio de almacenaje o cella vinaria, junto con dos salas más, también destinadas al almacenaje, anexo a lo cual se vislumbra un área dedicada posiblemente a vivienda que todavía queda por investigar. En este artículo se presenta un esgrafiado paleohispánico sobre cerámica Campaniense A, dos estampillas celtibéricas sobre tinaja y un esgrafiado latino en una vasija celtibérica hallados en las dependencias de este sitio arqueológico.

Palabras clave: Esgrafiado. Estampilla. Epigrafía paleohispánica. Epigrafía latina. Lagar.

Abstract: The Dehesa Cintruénigo III (Tarazona, Zaragoza) site corresponds to an old winery that includes a tread area on a trullo type lacus, the remains of a manual beam press of heronian typology and a storage space or cella vinaria, along with two more rooms, also for storage, annexed to which is an area possibly dedicated to housing that remains to be investigated. This paper consists of the publication of a Palaeohispanic grafitto on Campanian A pottery, two Celtiberian stamps on storage pottery and a Latin grafitto on a Celtiberian vessel found in this archaeological site

Key words: Grafitto. Stamp. Palaeohispanic Epigraphy. Latin Epigraphy. Winery.

Recepción: 08.10.2019 | Aceptación: 15.12.2019

Proyecto: La realización de este trabajo ha sido posible gracias a un contrato Juan de la Cierva (IJCI-2016-27589) y se inscribe en el proyecto FFI2015-63981-C3-3-P, "El final de las escrituras paleohispánicas”, financiado por el Ministerio de Economía y Ciencia y el Grupo Hiberus del Gobierno de Aragón. Las tres campañas de excavación en el yacimiento han sido costeadas por el área de Arqueología del Centro de Estudios Turiasonenses. 


\section{El yacimiento}

El yacimiento denominado "Dehesa Cintruénigo III" se encuentra ubicado casi equidistante ( $7 \mathrm{~km}$ aproximadamente) de las antiguas ciudades de Turiaso (Tarazona, Zaragoza) y Cascantum (Cascante, Navarra). Su situación está vinculada a una ruta natural que comunica la Meseta con el Valle del Ebro (fig. 1). En la actualidad este lugar cuenta con un paisaje compuesto por pequeños cabezos y barrancos que hoy día tienen un uso agropecuario de carácter extensivo. Además, su entorno se encuentra jalonado por asentamientos con una cronología que abarca desde el s. II a.C. hasta el s. V d.C. (García y Pérez 2011).
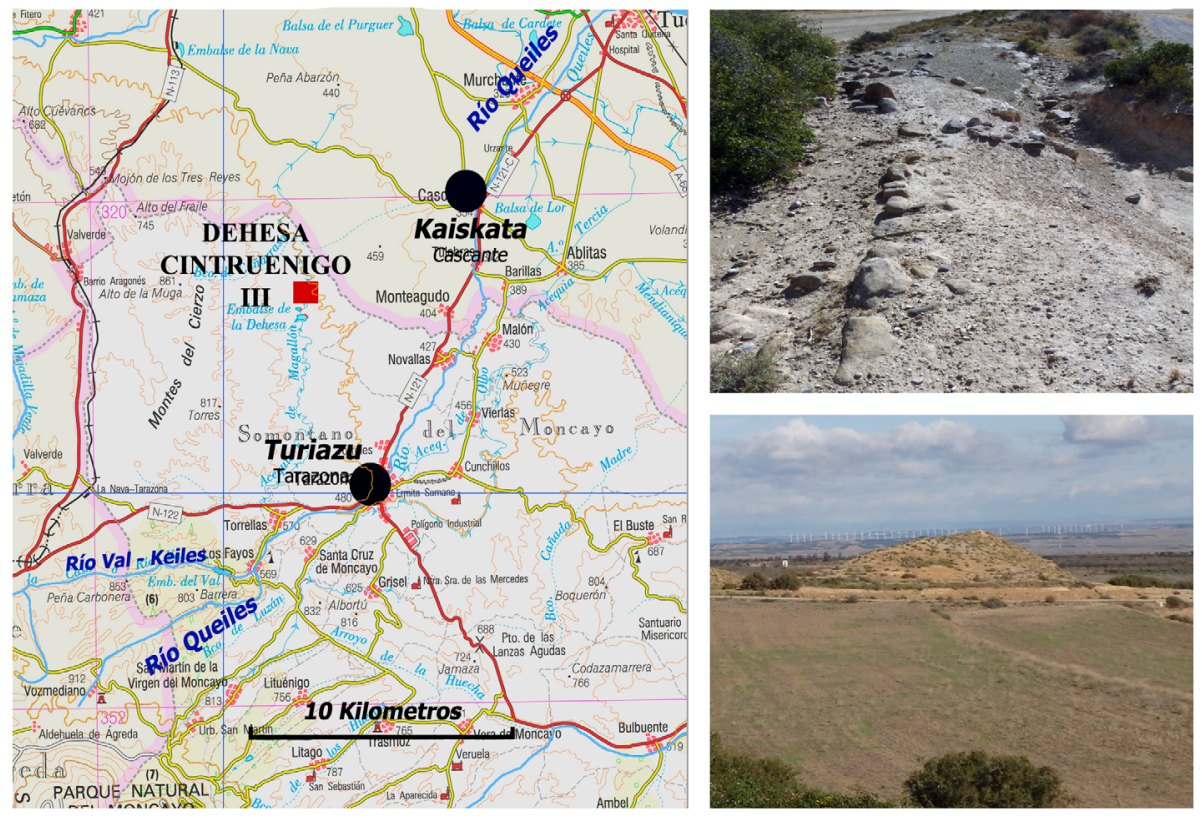

Fig. 1. Situación geográfica (izquierda), estado previo (derecha, arriba) y vista desde el sur (derecha, abajo) del yacimiento Dehesa Cintruénigo III.

La historia de las investigaciones en este yacimiento comienza en 2012 gracias a una prospección arqueológica realizada por el Centro de Estudios Turiasonenses (Pérez 2012). En esta intervención se documentaron, debido a una limpieza mecánica de un ramal de la acequia Magallón, y en la base de una pequeña loma no cultivada, una serie de vestigios arqueológicos, tanto muebles como inmuebles, que invitaron a pensar en una futura intervención de urgencia por parte de esta entidad debido a sus especiales características. Los materiales aparecidos fueron datados a finales del siglo II a.C. y principal- 
mente se identificaron pequeñas piezas de plomo cuadrangulares y rectangulares que, enrolladas sobre sí mismas, servirían como plomadas de redes de pesca en remanso, o bien, para la caza de pequeñas aves. Si bien, estos plomos ya no estaban en uso en aquel momento ya que muchos de ellos se encontraron desenrollados y, junto a ellos, además de cerámica de este horizonte temporal, también se documentó una torta de fundición, lo cual indicaba su reaprovechamiento mediante este proceso metalúrgico (García y Pérez 2015).

Tras esta primera investigación se han realizado tres campañas de excavación entre 2015 y 2017. A pesar de las primeras evidencias materiales, no se ha encontrado, por el momento, el lugar de fundición del plomo que era previsible descubrir, tal vez porque éste quedó destruido al construirse la acequia mencionada y el camino que da acceso a las fincas colindantes, lugar por el cual debió de continuar el emplazamiento, ahora desaparecido en su lado sur por completo. La sorpresa surgió al hallar un espacio de producción vitivinícola (figs. 2a-b) datado entre mediados del siglo II a.C. y el primer tercio del siglo I a.C., cuando esta instalación fue destruida a causa de un incendio (García, García y Pérez 2016). Este lagar cuenta con un lacus, de tipo trullo, asociado a la base de una prensa de viga manual de tipología heroniana. Junto a estos restos arquitectónicos se ha documentado en primer lugar una escalera que daba paso al lagar desde la zona no conservada y creaba el desnivel necesario para el tiro de la prensa; y en segundo lugar, la zona que se ha interpretado como el almacén o cella vinaria (fig. 2a), donde además se podría realizar el trasegado del vino; y dos salas más que siguen el perfil de lugar de almacenaje (figs. 2c-d), dados los ejemplos cerámicos encontrados en ellas, bien para vino, bien para otros productos agrícolas como pudiera ser el grano o su transformación en harina. Precisamente es en estos dos últimos espacios, $y$ en un tercero que ha sido muy parcialmente investigado (posiblemente el lugar de residencia), donde se han encontrado los esgrafiados y sellos que aquí estudiamos. La interpretación de este espacio como lagar ha sido corroborada con el análisis químico, con resultado positivo, para diversos elementos componentes del vino tinto (Blanco et al. 2019).

El yacimiento Dehesa Cintruénigo III supone, por lo tanto, una nueva aportación al conocimiento de los inicios de la viticultura en el ámbito celtibérico, dentro ya de un horizonte cultural celtíbero-romano ( $c f$. Burillo 2010a; 2010b). Además, los trabajos realizados en este yacimiento nos permiten constatar por primera vez un sistema de producción que optimiza los recursos existentes, al aprovechar una parte del propio lagar para el pisado de la uva. 
Esto se consigue insertando una estructura de madera a base de listones en la parte superior del lagar para pisar las uvas directamente en esa zona de manera que el zumo de la uva caería a través de las rendijas del entramado de madera al lacus (fig. 2b). Este modelo ha llegado hasta la actualidad con la denominación de trullo. La prensa del tipo heroniano (Heron, Pneum. III.2.1321) estaba situada a continuación y vertía también directamente al lagar (fig. 2a). De esta forma el acopio a la jaula de la prensa de los hollejos y raspones resultantes del pisado sería muy fácil (García, García y Pérez 2016, 395). El mosto resultante comenzaría una primera fermentación tumultuosa en el propio lacus durante algunos días. Más tarde sería trasegado manualmente a las tinajas de borde reentrante donde se produciría una segunda fermentación y su almacenamiento (fig. $2 b$ ).

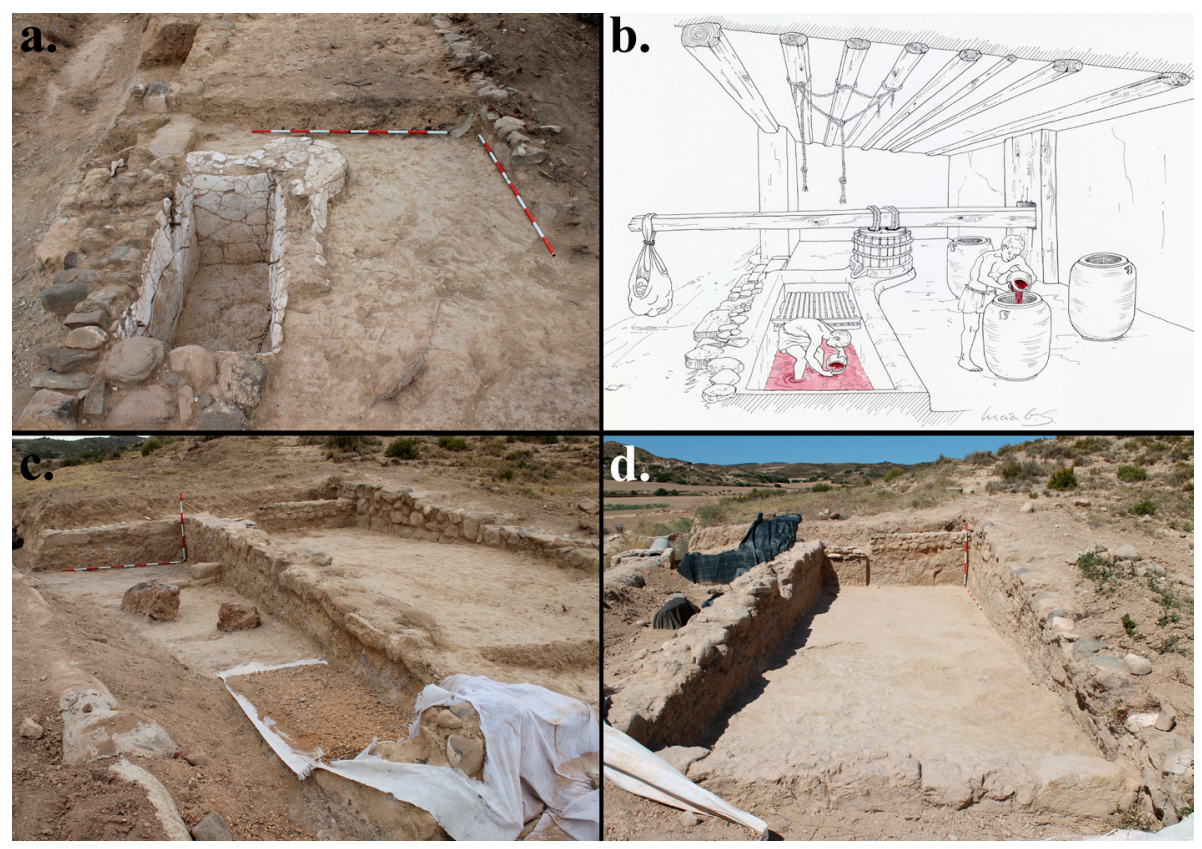

Fig. 2. Excavación en Dehesa Cintruénigo III:

a. Vista del lagar en 2015.

b. Reconstrucción hipotética del lagar (dibujo: L. Gómez Serra).

c. Zona de excavación en 2016.

d. Zona de excavación en 2017. 


\section{La epigrafía de Dehesa Cintruénigo III}

El territorio circundante del yacimiento que nos ocupa ha proporcionado una treintena de textos celtibéricos y latinos recientemente compilados en un estudio monográfico sobre el antiguo valle del Queiles (Beltrán y Simón 2017). ${ }^{1}$

Las excavaciones realizadas en Dehesa Cintruénigo III han proporcionado tres fragmentos de labio de tinaja estampillados (dos de ellos, con sellos monolíteros en escritura paleohispánica, y uno, anepígrafo), un grafito paleohispánico sobre cerámica Campaniense A y otro latino sobre una vasija de cerámica común. Pudimos realizar autopsia de todos ellos en el Centro de Estudios Turiasonenses, donde actualmente se conservan para su investigación hasta su depósito definitivo en el Museo de Zaragoza, el 4 de mayo de 2019.

\subsection{Las estampillas}

Los tres fragmentos de tinajas estampilladas se hallaron en el espacio 4, si bien, cada uno inserto en una UE diferente de las cuatro documentadas en esta sala. Este espacio se encuentra anexo al lagar pero no tiene comunicación con él. Su funcionalidad, gracias a los restos arqueológicos encontrados, apuntan a su identificación como una zona de almacenaje, quizás para vino, a pesar de la ausencia de una comunicación directa con la zona de producción, o bien para otro producto como pudiera ser grano, o su procesado en harina, ya que en esta habitación se encontró un molino circular, aunque este es un aspecto que todavía está en curso de estudio.

La estampilla 1 se descubrió en la UE10 del espacio 4. La UE10 se corresponde con el nivel de contacto con el suelo de esta habitación. La estampilla 2 se encontró en la UE8 del espacio 4. Esta UE se interpreta como una gran caída muraria, con dirección y pendiente este-oeste, que accedió a esta sala a través de la puerta que une los espacios 4 y 5 , seguramente arrastrando material cerámico de ambas habitaciones. La estampilla 3 se halló en la UE9 del espacio 4, que interpretamos como una mezcla de las caídas de las paredes de este espacio sobre sí mismo junto con los materiales cerámicos que había en su interior en los momentos del derrumbe.

1 Ver en el mismo volumen García, Pérez y García 2017 sobre la epigrafía latina y Amela 2017 sobre la numismática. Sobre la epigrafía y la lengua celtibéricas, ver el reciente volumen de Jordán 2019, cuyas páginas 349-419 están dedicadas a la epigrafía sobre instrumentum domesticum. 


\subsubsection{Estampilla 1}

El primer sello que presentamos fue impreso en un fragmento de tinaja tipo Ilduratin (fig. 3). La longitud máxima de la pieza son $29 \mathrm{~cm}$ y su anchura, $8 \mathrm{~cm}$; el labio mide $6 \mathrm{~cm}$ de ancho y tiene $1,2 \mathrm{~cm}$ de grosor, de pasta anaranjada y de interior ligeramente grisáceo, con desgrasantes muy finos, dura y suave al tacto. Aunque está realizada en cocción oxidante, sin embargo, su pasta grisácea interior señala un aporte distinto de oxígeno durante la cocción. El diámetro interno del borde mide $35 \mathrm{~cm}$ y el externo, $41,5 \mathrm{~cm}$. El grosor de sus paredes oscila entre 1 y $2,1 \mathrm{~cm}$. Este tipo de tinajas son habituales en los yacimientos celtibéricos del valle del Ebro desde el siglo IV al I a.C. (Burillo, Cano y Saiz 2008). En el territorio que nos ocupa estas producciones cerámicas pueden estar relacionadas con la fabricación local atestiguada en los alfares excavados en el sector 1 y 2 del oppidum de La Oruña (Sainz y Gómez 2008-09).

La estampilla fue impresa a $2,2 \mathrm{~cm}$ del borde izquierdo del fragmento, a $1 \mathrm{~cm}$ del borde superior y $4 \mathrm{~cm}$ del inferior, sin mucha profundidad. Fue grabada en posición interna y, en consecuencia, la base de la letra está en la parte interior de la vasija. El sello tiene forma cuadrada, $1,2 \mathrm{~cm}$ de lado, con los bordes redondeados y carece de decoración. Solo contiene una letra cuya altura máxima es $0,8 \mathrm{~cm}$ de altura y está ubicada en la parte inferior del cuadrado, dejando un amplio espacio en blanco en la parte superior. Está centrada con respecto de los laterales. Su trazo mide $1 \mathrm{~mm}$ de ancho.

El único grafema de este sello consiste en una $\mathbf{m} \mathbf{2}$ del signario celtibérico oriental. El trazo vertical está ligeramente torcido, y se une con la parte superior de la letra no en el centro, sino en uno de los trazos diagonales internos de $\mathbf{m}$. La variedad oriental del semisilabario celtibérico es coherente con la de otros grafitos paleohispánicos procedentes del mismo entorno (uid. p.ej. Beltrán y Simón 2017, nº 1, El Castellar de Fontellas; Royo 1978, de Borja; Jordán 2011, nº 5.8, también de Borja). Lectura: 

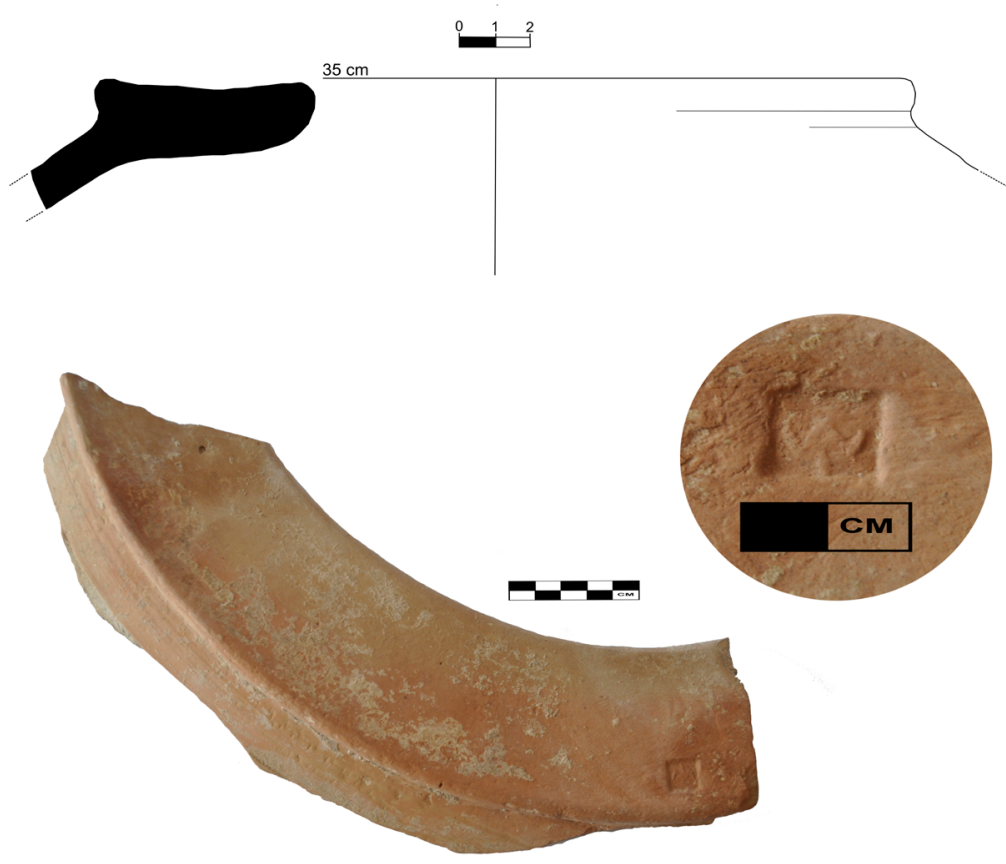

Fig. 3. Pieza 1 con estampilla.

\subsubsection{Estampilla 2}

El segundo sello fue impreso en un borde de tinaja tipo Ilduratin similar al anterior (fig. 4). En este caso se conserva parte del labio y el arranque de cuerpo globular donde se encuentra un asa triple fragmentada sobre la cual se observa el baquetón decorativo de la parte superior de la vasija. La longitud máxima de la pieza es $44,5 \mathrm{~cm}$ y su anchura máxima, $12 \mathrm{~cm}$. El labio mide, igual que el anterior, $6 \mathrm{~cm}$ de ancho y es más grueso: $2 \mathrm{~cm}$., el diámetro interno del borde tiene $31 \mathrm{~cm}$ y $37,2 \mathrm{~cm}$ la parte externa del mismo, junto con un grosor de sus paredes que oscila entre 1,1 y $2,2 \mathrm{~cm}$. Su pasta es marronácea, rugosa, poco depurada con desgrasantes visibles y de cocción oxidante.

La estampilla se selló a 12,5 cm del borde izquierdo y bien centrada: a $2 \mathrm{~cm}$ del borde superior y el inferior. Ésta fue impresa con mayor profundidad que la anterior, especialmente en su extremo inferior izquierdo, y en posición externa, al contrario que la estampilla 1 . El sello es ligeramente rectangular: $1,3 \mathrm{~cm}$ de alto por 1,1 de ancho. En este caso, los ángulos están más marcados que en el anterior. La letra que contiene mide $1,2 \mathrm{~cm}$, es decir, ocupa prácticamente toda la altura del campo epigráfico y toda la anchura. Su trazo tiene un grosor máximo de $2 \mathrm{~mm}$ y está roto en su extremo superior derecho, circunstancia que no impide la lectura. 
El signo que se representa en esta estampilla es una $\mathbf{n}$ del signario celtibérico oriental. Naturalmente, esta forma podría interpretarse como una $\mathbf{m}$ del signario celtibérico occidental; pero los testimonios coetáneos (uid. supra) y la compatibilidad con la estampilla 1 desaconsejan esta alternativa. En este caso, pues, se trataría de un alógrafo n1. Lectura:

n
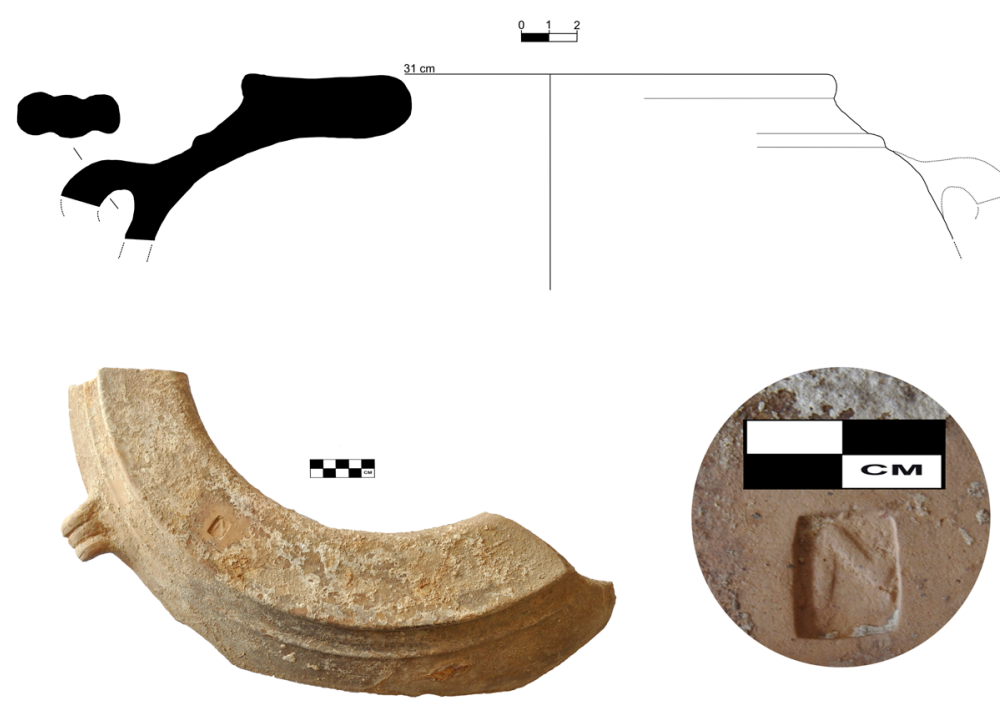

Fig. 4. Pieza 2 con estampilla.

\subsubsection{Estampilla 3}

Fragmento de borde de tinaja tipo Ilduratin, en el que fueron impresas dos estampillas superpuestas de características similares a las anteriores, cuyo contenido está completamente erosionado y resultan de todo punto ilegibles (fig. 5). El diámetro interno del borde es de $35 \mathrm{~cm}$ y $41 \mathrm{~cm}$ en su parte externa, con un grosor de sus paredes que oscila entre 1,4 y $2 \mathrm{~cm}$. Su pasta es anaranjada, ligeramente grisácea en el interior, con desgrasantes muy finos, dura y suave al tacto. Está realizada en cocción oxidante; sin embargo, también muestra pasta grisácea en el interior lo que señala un aporte distinto de oxígeno durante la cocción. 


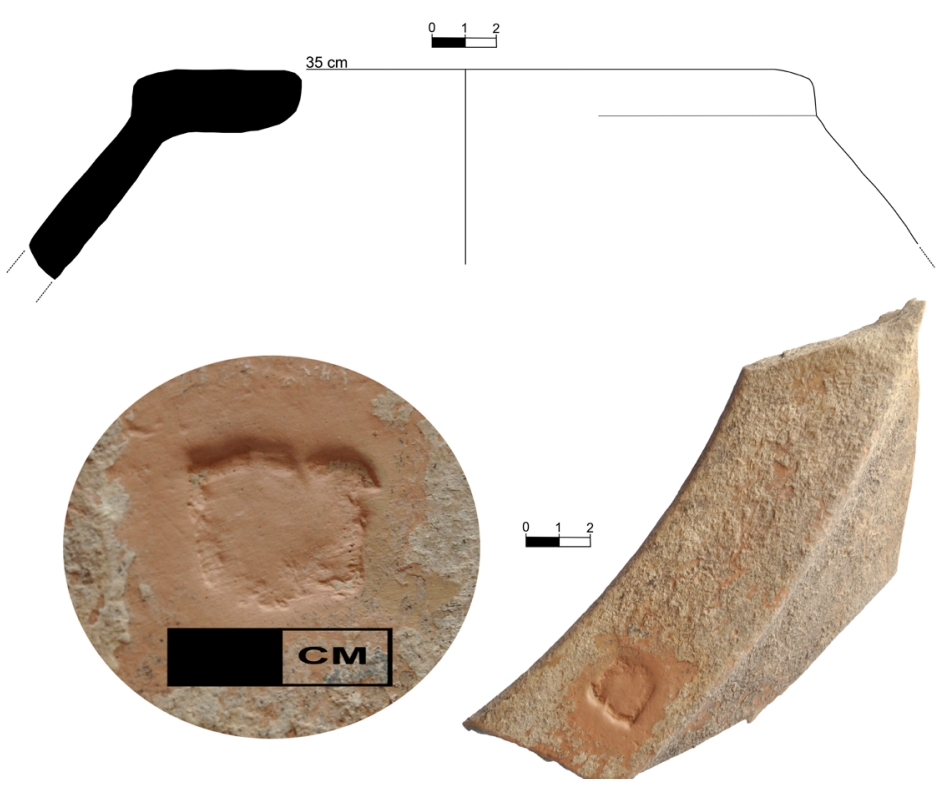

Fig. 5. Pieza 3 con estampilla doble.

\subsubsection{Las inscripciones paleohispánicas sobre tinajas}

Las estampillas paleohispánicas están documentadas principalmente en pesas de telar y en recipientes de almacenaje, preferentemente, tinajas y, en menor medida, ánforas ( $c f$. Salvat 2005; Simón 2008; 2013, 565). Este particular tipo de textos se han recuperado mayoritariamente en el sur de Francia (principalmente, en Ensérune), la costa catalana y el valle del Ebro, es decir, en ámbito ibérico. A cambio, el elenco de estampillas en ámbito celtibérico, no necesariamente escritas en lengua celtibérica, ${ }^{2}$ es más escaso: se han recuperado seis sellos sobre este tipo de cerámica de almacenaje (tabla 1).

2 El hallazgo de las estampillas en la región celtibérica no implica necesariamente que éstas estuvieran escritas en lengua celtibérica: el ejemplar de El Piquete de la Atalaya es el que se ha postulado con más seguridad en este sentido (cf. Burillo 1993-1995, MLH K.21.1, Simón 2013, 595). Por lo que respecta a bilonike, de Contrebia Belaisca, podría ser un nombre griego adaptado al ibérico ( $M L H$ IV, 609). Según I. Simón, el tipo de escritura de esta podría ser levantino (Simón 2013, ED28 propone, con dudas, esta posibilidad), lo que encajaría bien con el final en -e que se ha identificado para los nombres latinos de la epigrafía ibérica como bilake o likine.

El resto son tan breves que no es posible deducir de ellos ninguna adscripción lingüística, si bien es cierto que los textos monolíteros o bilíteros en las estampillas sobre dolios como los que presentamos aquí solo se circunscriben a la Celtiberia y sus aledaños septentrionales (Simón 2013, ED30, ED31, ED32; Jordán 2019, 349-419). 


\begin{tabular}{|c|c|c|c|}
\hline Referencias & Texto & Tipo de contenido & Lugar de hallazgo \\
\hline BDH Z.09.07 & bilonike & antropónimo & Contrebia Belaisca \\
\hline$B D H$ Z.14.01 & memo $\cdot$ bel & antropónimo + origo & Azuara \\
\hline BDH LO.05.02 & ska (o kas) & $?$ & Contrebia Leucada \\
\hline BDH NA.02.01 & ka (o $A)$ & $?$ & Los Arcos \\
\hline BDH NA.01.11 & rl & $?$ & La Custodia \\
\hline Labeaga 1999-00 & tîs (monograma) & $?$ & La Custodia \\
\hline
\end{tabular}

Tabla 1. Estampillas sobre cerámica procedentes del ámbito celtibérico.

Paralelos claros de las estampillas que presentamos aquí son las de Los Arcos, Contrebia Leucada y La Custodia por varias razones. En primer lugar, todas ellas están situadas en el labio del dolio, a diferencia de los ejemplares ibéricos del sur de Francia o los propiamente romanos, en los que los sellos aparecen bajo el borde y no sobre él (Luezas 1998, 218). ${ }^{3}$ Además, los sellos de Dehesa Cintruénigo III carecen de gráfila o cualquier otro tipo de decoración como las que puedan tener las estampillas catalanas (Simón 2013, ED24, ED25, ED26. Salvo los dos puntos que se aprecian en la estampilla de Los Arcos, Simón 2013, ED32). Tercero, la cartela de estos sellos es cuadrangular, como la de Los Arcos, y de esquinas tendentes a redondeadas, como la de Contrebia Leucada (aunque su tamaño es un poco menor). De hecho, formalmente, las estampillas de Dehesa Cintruénigo III no distan mucho de algunos sellos impresos sobre pesas de telar (Simón 2013, 645): misma forma cuadrangular, esquinas redondeadas, tamaño similar (uid. p.ej. Simón 2013, EP3 y EP10). Y, por último, todas ellas son estampillas monolíteras o bilíteras. A diferencia de los sellos de Azuara y Botorrita, que contienen un antropónimo completo o una fórmula antroponímica abreviada, posiblemente referidos al fabricante del recipiente, el resto de estampillas consisten en secuencias bilíteras que bien podrían ser una abreviatura del nombre de un fabricante o bien, tener otra naturaleza.

En realidad no conocemos con precisión el significado de los textos de las estampillas paleohispánicas y los paralelos que proporciona la epigrafía latina

3 Una ubicación en la que no estarían muy visibles, si se tiene en cuenta que probablemente estos dolios estarían tapados (Simón 2013, 594), como sugiere una tapa de dolio hallada en el mismo yacimiento de Dehesa Cintruénigo III. 
sobre dolia de la península Ibérica no es de mucha ayuda: en la nómina de Salido (2017, 281-289) apenas dos dolios son fechables en época republicana: Flaccini (Luezas 1998, 217-218, de Contrebia Leucada), y MVA (Caballero 1992, 254, de Arcobriga), éste último en una tinaja de tipo Ilduradin, como las que nos ocupan, fechable de la segunda mitad del siglo II a.C. en adelante. Los textos de los sellos de época imperial son más largos.

Cualquier interpretación de esta pareja de sellos monolíteros no supera el nivel hipotético; aunque nos inclinamos por pensar que estamos ante sendos marcadores de referencia de una producción determinada de dolia, una información interna de la figlina, consistentes en letras sueltas, principalmente porque se trata de una información que no era especialmente visible en el ámbito del lagar $y$, además, porque la parte superior de la tinaja posiblemente estaba cubierta.

\subsection{Grafito paleohispánico}

Ejemplar casi completo de cuenco en cerámica de Campaniense A tardía, con una datación de c. 100-40 a.C., forma Lamb. 31b / Morel 2950, (fig. 6). El cuenco, de $16 \mathrm{~cm}$ de diámetro de borde, $5,5 \mathrm{~cm}$ de diámetro externo y 3,5 de diámetro interno en su base, $0,9 \mathrm{~cm}$ de grosor en su pie y una altura total de $7,7 \mathrm{~cm}$. Realizado a torno, posee una superficie rugosa al tacto con estrías. Su pasta es de color marrón-rojizo con partículas de mica, bastante dura y granulosa. El barniz negro está bastante degradado y diluido debido a su aplicación por inmersión. En el interior presenta decoración formada por una línea blanca bajo el borde interno y dos círculos no concéntricos en el fondo, también en el fondo conserva la marca circular en el engobe interno que se produce durante el apilamiento de las piezas en el proceso de cocción. Se recuperó en el Espacio 2 dentro de la UE6. Esta UE se interpreta como un nivel de destrucción por parte del fuego que consumió parte de esta instalación en su zona productiva como lagar (García, García y Pérez 2016), y que contenía restos cerámicos de grandes contenedores, junto a otros de vajilla de menor tamaño.

Dos signos fueron incisos después de la cocción, dentro del pie, de forma profunda, dejando ver la pasta anaranjada. Aunque se conserva claramente el barniz de color negro, éste se ha desconchado en muchos puntos, cosa que podría influir en la lectura del grafito. La altura máxima de estas letras es $0,9 \mathrm{~cm}$ en el caso de la letra 1 y 0,6 cm en el de la letra 2 . 
La escritura presenta un sentido dextrorso, a juzgar por el primer grafema, $\mathbf{1 1}$ celtibérico / $\mathbf{1 2}$ ibérico. Después de realizar la autopsia al grafito, hemos descartado la posibilidad de que se trate de un signo ki ya que el arranque de un hipotético trazo diagonal en la parte superior es solo aparente y se trata de un desconchón del barniz al introducir el punzón con el que se grabó el grafito.

El segundo grafema plantea una interpretación más complicada, ya que consiste en un gran trazo curvo y otro rectilíneo. Descartamos que el punto interior tenga una intención grafemática, ya que en la superficie de todo el pie se aprecian abundantes saltos del barniz que dejan ver el color naranja de la pasta (aunque, vistas las posibilidades de lectura y sus posibles paralelos, no habría que descartarlo). Además, creemos que el trazo rectilíneo podría ser fruto de las dificultades que pudiera tener el incisor en un campo epigráfico tan menguado, y que su intención sería grabar un círculo, que hizo en cuatro tiempos, como es claramente visible en los trazos de este esgrafiado. Este grafema podría estar expresando un valor ku (ku1 o ku3 celtibérica; ku3 ibérico) o r/ŕr (r3 o r7 celtibérica; ́r 1 o ŕ8 ibérica). En consecuencia, proponemos la siguiente lectura:

\section{lr o lku}
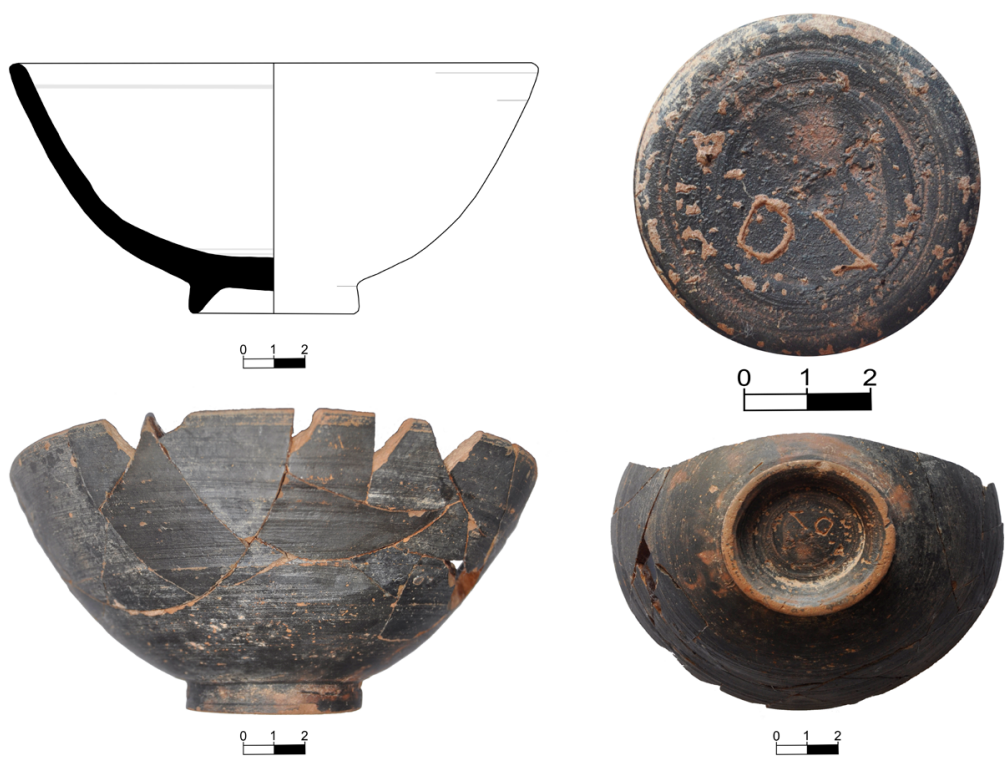

Fig. 6. Pieza 4 con esgrafiado paleohispánico. 
Cualquiera de las dos secuencias propuestas para la lectura de este grafito, Ir o lku, aparece con cierta frecuencia en la epigrafía paleohispánica sobre cerámica, tanto en el ámbito celtibérico como en el ibérico.

En el primero está documentada en un sello sobre dolio de La Custodia, ya mencionado. Además, esta secuencia es relativamente abundante en Contrebia Belaisca y en Contrebia Leucada. Por lo que respecta a Botorrita, rl uel sim. comparece en un esgrafiado sobre un kalathos (MLH IV K.1.8), sobre una campaniense ( $M L H$ IV K.1.9), inciso en una pesa de telar (MLH IV K.1.23) y sobre un thimiaterion (Díaz y Jordán 2001, 313 y 329). En el corpus de Contrebia Leucada estos dos grafemas están presentes en varios esgrafiados y en una pareja de estampillas. ${ }^{4}$

En el ibérico, esta pareja de grafemas está atestiguada, por ejemplo, en dos pondera de Azaila (MLH III E.1.419), para la que Untermann propuso una lectura lŕ o lku y en dos grafitos sobre campaniense del mismo yacimiento (E.1.156, kulki, y E.1.157, kul). Asimismo, también lo está en una campaniense de Baetulo (Panosa 1993, 190, n 11.4). Como kul han sido leídos también el grafito sobre campaniense de Ensérune $M L H$ II B.1.97; un grafito también sobre cerámica de barniz negro procedente de Lérida (Garcés y Sabaté 2017, 245-246, no 10) y otro sobre cerámica de los alrededores de Amposta (Panosa 2015, 62-63). En todos estos casos, la lectura de 1 parece segura. En cambio, la lectio del segundo grafema es más complicada; aunque hay casos en los que claramente es una vibrante y otros, en los que no hay duda sobre su transcripción ku (cf. tabla 2).

4 Agradecemos sinceramente a C. Jordán, I. Simón y J. A. Hernández Vera que nos hayan informado de la existencia de estas inscripciones. 


\begin{tabular}{|c|c|c|c|}
\hline referencia & tipo de inscripción & texto & lugar de hallazgo \\
\hline Labeaga 1999-00. & sello sobre dolio & $\mathbf{r l}^{5}$ & La Custodia \\
\hline MLH IV K.1.9. & grafito sobre cerámica & rl & Contrebia Belaisca \\
\hline MLH IV K.1.23. & grafito sobre pondus & kul o ŕl & Contrebia Belaisca \\
\hline MLH IV K.1.8. & grafito sobre kalathos & rl & Contrebia Belaisca \\
\hline Díaz y Jordán 2001, n. ${ }^{\circ} 23$ & grafito sobre thimiaterion & lr & Contrebia Belaisca \\
\hline Beltrán 1995, 188 & grafito sobre pondus & lku & Azaila \\
\hline MLH III E.1.419. & grafito sobre pondus & lí o lku & Azaila \\
\hline MLH III E.1.156. & grafito sobre campaniense & kulki & Azaila \\
\hline MLH III E.1.157. & grafito sobre campaniense & kul & Azaila \\
\hline MLH II B.1.97. & grafito sobre campaniense & kul & Ensérune \\
\hline Panosa 2015, 62-63. & grafito sobre campaniense & kul[---] & Amposta \\
\hline Panosa 1993, 190. & grafito sobre campaniense & lí o lku & Baetulo \\
\hline Garcés, Sabaté 2017, n.⒑ & grafito sobre campaniense & kul & Lérida \\
\hline inéditos & grafitos y sellos sobre cerámica & lku & Contrebia Leucada \\
\hline
\end{tabular}

Tabla 2. Secuencias lr, lí, lku y kul en inscripciones sobre cerámica ibéricas y celtibéricas.

No hay motivos claros que permitan adscribir cada una de las dos posibilidades de lectura (lku o lr y sus variantes) a un ámbito cultural concreto, ibérico o celtibérico. Si bien es cierto que la mayor parte de los testimonios de la zona celtibérica apuntarían hacia una lectura lr o rl y descartar la posibilidad con ku, al tener con un trazo vertical en todos los casos, el pondus de Contrebia Belaisca y, sobre todo, la documentación inédita de la Leucada, nos hacen pensar en una lectura lku. Por lo que respecta a la documentación del área ibérica, parece que todos los testimonios salvo uno de los pondera de Azaila contienen una marca en el centro del grafema romboidal (punto o raya), de modo que habría que decantarse por la lectura ku. Además, es oportuno recordar que todos los objetos que contienen esta secuencia son susceptibles de ser transportados fácilmente a un lado y al otro de las fronteras culturales paleohispánicas. En conclusión, podríamos afirmar que la adscripción cultural ibérica o celtibérica del lugar de hallazgo no nos permite decantarnos con seguridad por la lectura lr o por la lectura lku.

5 Este sello elíptico contiene dos grafemas enlazados: $\mathbf{r}$ y l, alógrafos $\mathbf{r} \mathbf{4}$ y $\mathbf{1 2}$ celtibérico. En el dibujo de Labeaga 1999-00, fig. 536 se aprecia claramente un pequeño trazo que permite distinguir el grafema $\mathbf{r}$ de ku. En la publicación en curso de los grafitos de Contrebia Leucada, que muy amablemente nos han dejado consultar los autores, se documenta un sello muy similar a éste cuya lectura es, inequívocamente, rl. 
Por lo que respecta al significado, es muy arriesgado aventurar cuando se trata de documentos tan breves, y más teniendo en cuenta que estas marcas comparecen tanto en ámbito ibérico como celtibérico, tanto en sellos como en esgrafiados, escritos sobre un variado abanico de soportes cerámicos (campanienses, kalathoi, thimiateria, pondera, dolia), suponiendo, claro está, que todos tengan el mismo referente. En algunos casos anteriormente recogidos en los que los editores leen kul, se propone que se trate de una abreviatura del antropónimo kule(ś) como marca de propiedad (p.ej. Panosa 1993, 63). Sin embargo, cuando se trata de las secuencias lku, rl o lr, difícilmente se podría defender esta marca como la abreviatura de un antropónimo: cualquiera de las combinaciones tiene difícil encaje como tal tanto en ibérico como celtibérico.

La frecuencia de la pareja de caracteres r o ku y $\mathbf{l}$ es llamativa en la epigrafía 'menor' paleohispánica, por lo que podría elucubrarse que podríamos estar ante una marca propia de los recipientes cerámicos cuyo significado se nos escapa, o una marca de valor, o quizá símbolos numerales, opción que quedaría avalada, quizá, por el hecho de que ku es una bien atestiguada leyenda secundaria en la numismática celtibérica e ibérica ( $c f$. García-Bellido 1989; Estarán 2013, 70). La reiteración en las secuencias incisas en los grafitos es un hecho bien conocido, por ejemplo, en los conjuntos de Azaila o de Numancia (Arlegui 1992). En este caso, la marca sería visible cuando el recipiente estuviera vacío y apilado boca abajo, ocupando la parte superior de la pila.

\subsection{Esgrafiado latino}

Ejemplar casi completo de jarrita de cerámica común celtibérica de un tipo bastante extendido en el valle del Ebro, con una amplia cronología de uso entre el s. III y el I a.C. (Burillo, Cano y Saiz 2008), y encuadrada dentro la vajilla de servicio para la manipulación de líquidos. La pieza está formada por un conjunto de fragmentos que constituyen una forma carente de fondo, con borde exvasado, perfil carenado y asa vertical doble con acanaladuras. Tiene una altura máxima conservada de $14,5 \mathrm{~cm}$ y $0,4-0,7 \mathrm{~cm}$ de grosor en sus paredes. Se ha podido calcular un diámetro en su boca de $18 \mathrm{~cm}$. Se realizó a torno y consta de una pasta anaranjada con cocción oxidante en la que se pueden observar vacuolas o fallos de cocción y algún desgrasante macroscópico en superficie. Se recuperó en el Espacio 5 dentro de la UE7 (fig. 7). Esta UE, debido a la parquedad de datos que de ella se tienen (ya que únicamente se accedió a ella para delimitar el muro que compartimentaba los espacios 4 y 5), no ha sido interpretada por el momento adecuadamente, aunque sí que 
podemos decir que contiene en su mayoría restos de cerámica fina y de mesa, muy al contrario que el resto de UEs extraídas de este yacimiento. Por este motivo y otros, pensamos que esta zona pudo ser la parte residencial de este pequeño enclave.

El texto, grabado después de la cocción con muy poca profundidad, mide $3,7 \mathrm{~cm}$ de largo. Se conserva íntegro. Sus seis letras, miden entre 0,9 y 1,5. El texto está escrito en alfabeto latino, con tendencias cursivas: el trazo interior de $A$ es vertical y arranca desde el centro del trazo diagonal derecho. La $P$ presenta el bucle abierto, lo que apunta a una cronología de época republicana. La lectura es clara:

\section{Parati}

Parati podría interpretarse de dos formas distintas: bien, como un nombre personal masculino en genitivo singular o bien, en clave de interacción con los lectores, como una exhortación a estar 'preparados' para recibir la bebida que contiene la jarra si, como el contexto apunta, se trataba de vino. Paratus es un nombre bien documentado en la epigrafía latina de prácticamente todo el imperio ( $c f$. Kajanto 1982, 260). En la Hispania Citerior está atestiguado en la estela funeraria de Cecilia Parata procedente de Hontanar (HEp 1994, 888); en la tabula patronatus de Sasamón (CIL II 5812) y en un sello de sigillata procedente de Tarraco (CIL II 4970,5).

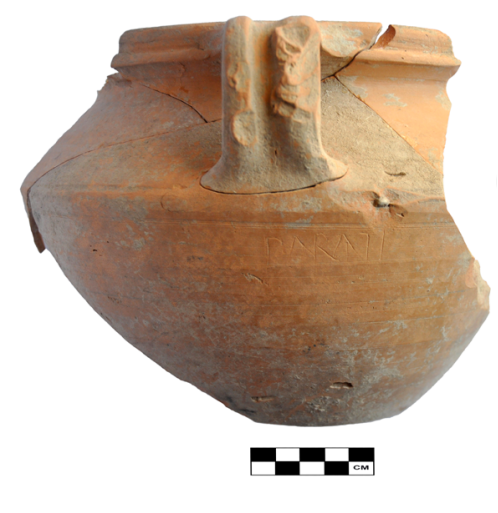

Fig. 7. Pieza 5 con esgrafiado latino.
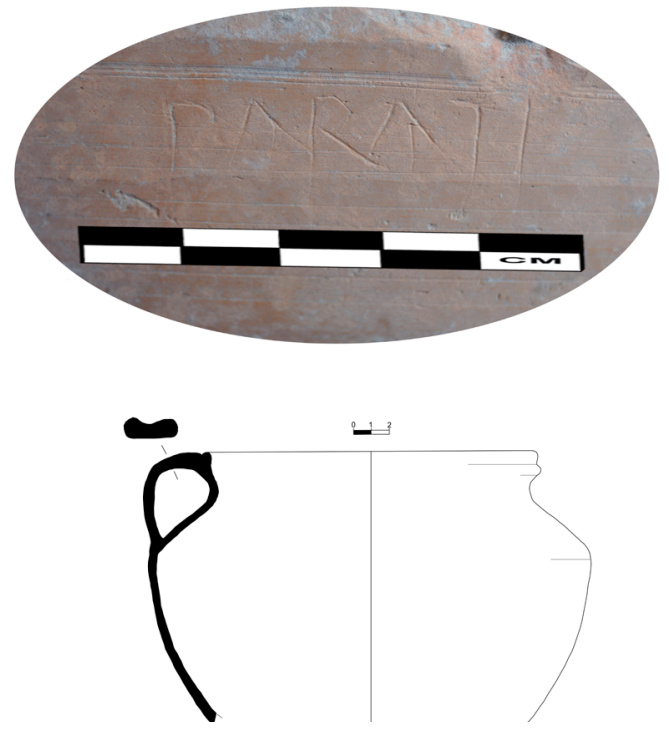


\section{Conclusión}

Los cuatro textos que se han presentado aquí vienen a engrosar el corpus de treinta inscripciones halladas en el valle del Queiles ( $c f$. Beltrán y Simón 2017). Las estampillas contribuyen a confirmar que la práctica del marcado de tinajas en la Celtiberia estaba, quizá, más extendida de lo que a priori podría parecer. Como se ha señalado, no es fácil determinar con precisión a qué hacen referencia las letras impresas en dichos sellos, aunque, dado que se imprimieron sobre la arcilla fresca, probablemente se refieran a marcas de control de la producción alfarera, de hecho, es interesante señalar que $\mathbf{m}$ y n, también hayan sido incisas en la boca de otros dolia (Jordán 2019, 377378). Estas marcas dejarían de perder su utilidad una vez estuvieran ya en uso, como en este caso, destinadas al almacenaje de vino o de grano.

El grafito paleohispánico, aunque breve, nos ha puesto sobre la pista de dos secuencias muy frecuentes en la epigrafía paleohispánica del nordeste peninsular, sea en el ámbito lingüístico ibérico o en el celtibérico: $\mathbf{l r} / \mathbf{r l}$ (o la variante con ŕ ) y lku/kul. Presente en una amplia variedad de soportes, posiblemente se trate de marcas numéricas y no de propiedad; aunque nos encontramos en un estado de la investigación probablemente un poco precoz para emitir una conclusión firme sobre su significado. Este grafito, que apareció con otras cerámicas de uso habitual, era visible una vez el cuenco estaba apilado boca abajo.

La inscripción latina de la jarrita, hallada en lo que posiblemente fuera un ambiente de vivienda, puede considerarse una marca de propiedad. La interpretación más razonable es que se trate de un nombre personal en genitivo que podría traducirse como "de Parato". La cronología de esta pieza, avalada por el contexto arqueológico, la tipología cerámica y la paleografía confirma que estamos ante una de las inscripciones latinas más antiguas de esta zona, quizá la única datable en el periodo republicano, momento en el que también pueden fecharse las otras tres inscripciones paleohispánicas que presentamos aquí, lo que contribuye a pensar que el latín comenzó a implantarse relativamente temprano, al menos a juzgar por los documentos con los que contamos. No es de extrañar: la ciudad emitió leyendas monetales en alfabeto latino ya en el 29 a.C. 


\section{$\begin{array}{|lllllllllllll|}\text { | } & \text { B } & \text { I } & \text { B } & \text { L } & \text { I } & \text { O } & \text { G } & \text { R } & \text { A } & \text { F } & \text { I } & A\end{array}$ |}

Arlegui 1992: M. Arlegui, "Las cerámicas de Numancia con letrero ibérico", en: II Symposium de Arqueología Soriana: homenaje a Teógenes Ortego y Frías. Vol. 1, Soria 1992, 473-494.

Armendáriz 2006: J. Armendáriz, “Bases arqueológicas para la localización de la ciudad vascona de Curnonium en Los Arcos (Navarra)", TAN 19, 2006, 85-108.

Beltrán y Simón 2017: F. Beltrán e I. Simón, “La epigrafía del valle del Queiles”, en: C. García, J. Á. García y J. Pérez (eds.), Arqueología y poblamiento en el valle del Queiles, Tarazona 2017, 191-205.

Beltrán, M. 2004: M. Beltrán, “Turiaso. La ciudad romana”, Caesaraugusta 76 (2004), 23-31.

Beltrán, M. 2013: M. Beltrán, Azaila. Estado de la cuestión en el año 2013 (contiene documentación inédita de Juan Cabré), Zaragoza 2013.

Blanco et al. 2019: L. Blanco, M. Olivares, K. Castro, J. A. Carretero, C. García, J. Á. GarcíaSerrano, J. Pérez, J. Pérez, "Wine markers in archeological potteries: detección by GC-MS at ultratrace levels", Analytical and Bioanalytical Chemistry 411, 2019, 6711-6722.

Burillo 2010a: F. Burillo, "Vino y ritual en La Celtiberia”, en: F. Burillo (ed.), VI Simposio sobre los celtíberos, Ritos y mitos, 573-594, Daroca 2010, 573-594.

Burillo 2010b: F. Burillo, "La vid y el vino en el valle medio del Ebro durante la etapa prerromana”, en: De la cuina a la taula, IV Reunió d'economia en el primer mil $\times$ leni a.C., Valencia 2010, 140-141.

Burillo, Cano y Saiz 2008: F. Burillo, M. A. Cano y M. E. Saiz, "La cerámica celtibérica”, en: D. Bernal y A. Ribera (eds.), Cerámicas hispanorromanas: un estado de la cuestión, Cádiz 2008, 171-187.

Caballero 1992: L. Caballero, Arcóbriga II. Las cerámicas romanas, Zaragoza 1992.

Díaz y Jordán 2001: M. A. Díaz, C. Jordán, "Grafitos procedentes de Contrebia Belaisca", PalHisp 1, 2001, 301-333.

Estarán 2013: M. J. Estarán, "Epigrafía monetal paleohispánica. Las leyendas secundarias", PalHisp 13, 2013, 65-83.

Garcés y Sabaté 2017: I. Garcés y V. Sabaté, "Nous esgrafiats ibèrics i llatins d'Ilerda (Lleida)", RAP 27, 2017, 237-265.

García-Bellido 1989: M. P. García-Bellido, "Las marcas de valor en las monedas celtibéricas", Gaceta Numismática 94-95, 1989, 55-64.

García, García y Pérez 2016: C: García, J. Á. García, J. Pérez, “Contribución al conocimiento de la elaboración del vino en el ámbito celtíbero-romano. Dehesa Cintruénigo III (Tarazona, Zaragoza)", en: J. I. Lorenzo y J. M. Rodanés (eds.), I Congreso arqueología y patrimonio aragonés, Zaragoza 2015, 389-397.

García y Pérez 2011: J. Á. García, J. Pérez, "El poblamiento rural romano en el área de influencia del Municipium Turiaso. Patrones de asentamiento en torno al río Queiles, término municipal de Novallas", Turiaso 20, 2011, 55-95.

García y Pérez 2015: J. Á. García y J. Pérez, "Algunos aspectos poco conocidos de la economía rural doméstica en el entorno celtíbero-romano de Turiazu (Tarazona, Zaragoza): La pesca y el reciclaje del plomo", Turiaso 22, 2015, 37-66.

García, Pérez y García 2017: J. Á. García, J. Pérez, C. García, “Corpus de inscripciones romanas en el valle del Queiles”, en: C. García, J. Á. García y J. Pérez (eds.), Arqueología y poblamiento en el valle del Queiles, Tarazona 2017, 263-304.

Jordán 2011: C. Jordán, “Chronica Epigraphica Celtiberica VI”, PalHisp 11, 2011, 285-318. 
Jordán 2019: C. Jordán, Lengua y epigrafía celtibéricas, Zaragoza 2019.

Kajanto 1982: I. Kajanto, The Latin Cognomina, Roma 1982.

Labeaga 1999-00: J. C. Labeaga, La Custodia, Viana. Vareia de los Berones, Pamplona 1999-00.

Luezas 1998: R. A. Luezas, "Sellos de alfarero sobre dolia en la Península Ibérica”, Caesaraugusta 74, 1998, 207-233.

Panosa 1993: M. I. Panosa, "Nuevas inscripciones ibéricas de Cataluña”, Complutum 4, 1993, 175-222.

Panosa 2015: M. I. Panosa, Inscripcions ibèriques de les comarques de Tarragona, Tarragona 2015.

Paracuellos, Pérez y García 2005-07: P. A. Paracuellos, A. J. Pérez, J. Á. García, “Cañada Madre. Un nuevo yacimiento romano republicano en la comarca de Tarazona y el Moncayo", Turiaso 18, 2005-07, 215-234.

Pérez 2012: M. C. Pérez Omeñaca, Prospección arqueológica y actualización de yacimientos en los términos municipales de Grisel, Litago, Novallas, Tarazona y Trasmoz (Zaragoza). Inédito. Exp. 135/2012.

Royo 1978: J. I. Royo, "La cerámica campaniense en Bursau", Cuadernos de Estudios Borjanos $1,1978,17-28$.

Sainz y Gómez 2008-2009: M. E. Saiz y S. Gómez, "Avance del estudio de la alfarería celtibérica en la Oruña (Vera de Moncayo-Trasmoz, Zaragoza)", Turiaso 19, 2008-09, 35-62.

Salido 2017: J. Salido, "Los dolia en Hispania. Caracterización, funcionalidad y tipología", en: C. Fernández, A. Morillo y M. M. Zarzalejos (eds.), Manual de cerámica romana III: cerámicas romanas de época altoimperial III: cerámica común de mesa, cocina y almacenaje, imitaciones hispanas de series romanas, otras producciones, Madrid 2017, 237-309.

Salvat 2005: E. Salvat, "La técnica del estampillat a l'epigrafia ibèrica i celtibérica", Sylloge Epigraphica Barcinonensis 5, 2005, 149-171.

Simón 2008: I. Simón, “Dos estampillas inscritas sobre pesas de telar de la colección Samitier”, PalHisp 8, 2008, 257-278.

Simón 2013: I. Simón, Los soportes de la epigrafía paleohispánica, Zaragoza-Sevilla 2013.

Simón 2017: I. Simón, “Corpus de inscripciones paleohispánicas del valle del Queiles", en: C. García Benito, J. Á. García y J. Pérez (eds.), Arqueología y poblamiento en el valle del Queiles, Tarazona 2017, 305-315. 